Pacific

Journal of

Mathematics

ON THE EXTREMAL FUNCTIONS OF SOBOLEV-POINCARÉ INEQUALITY

Meisun ZHu

Volume $214 \quad$ No. 1

March 2004 


\title{
ON THE EXTREMAL FUNCTIONS OF SOBOLEV-POINCARÉ INEQUALITY
}

\author{
Meijun Zhu \\ Dedicated to Elliott H. Lieb on the occasion of his 70th birthday
}

We prove the existence of extremal functions of SobolevPoincaré inequality on $S^{n}$ for $p \in(1,(1+\sqrt{1+8 n}) / 4)$. For general $n$-dimensional compact Riemannian manifolds embedded in $R^{n+1}$, such an existence result is proved for $p \in(n /(n-$ $1),(1+\sqrt{1+8 n}) / 4)$.

\section{Introduction.}

Let $\left(M^{n}, g\right)$ be a $n$-dimensional compact Riemannian manifold without boundary. The standard Sobolev-Poincaré inequality can be stated as the following: For any $p \in[1, n)$, there is a constant $A\left(p, M^{n}, g\right)>0$ such that

$$
\left(\int_{M^{n}}\left|u-u_{a}\right|^{p_{*}}\right)^{p / p_{*}} \leq A\left(p, M^{n}, g\right) \int_{M^{n}}\left|\nabla_{g} u\right|^{p}, \quad \forall u \in W^{1, p}\left(M^{n}\right)
$$

where $u_{a}=\frac{1}{\operatorname{vol}\left(M^{n}\right)} \int_{M^{n}} u, p_{*}=n p /(n-p)$ is the Sobolev conjugate of $p$. This inequality can be proved by combining Sobolev inequality with Poincaré inequality, see, for example, Hebey's book [8]. In this paper we are interested in the estimates of the best constant and the existence of extremal functions to the above inequality. Analytically these are naturally motivated questions. On the other hand they may have interesting geometric implications, in particular, in the study of Poincaré's isoperimetric inequality. We shall discuss this geometric issue in another paper [9].

Generally speaking, the existence of extremal functions is not a trivial issue, given the fact that $p_{*}$ is the critical Sobolev exponent for the Sobolev embedding theorem. In this paper, for a model manifold -the unit sphere $S^{n}$ with the standard metric $g_{0}=\sum_{i=1}^{n+1} d x_{i}^{2}$, we obtain a fairly satisfying result. Define the Sobolev-Poincaré quotient by

$$
I_{p}(u):=\frac{\int_{S^{n}}|\nabla u|^{p}}{\left(\int_{S^{n}}\left|u-u_{A}\right|^{p_{*}}\right)^{p / p_{*}}}
$$

where $u_{A}=\frac{1}{\operatorname{vol}\left(S^{n}\right)} \int_{S^{n}} u$. We are going to prove: 
Theorem 1.1. If $1<p<(1+\sqrt{1+8 n}) / 4$, then

$$
P_{\mathrm{I}}\left(S^{n}, p\right):=\inf \left\{I_{p}(u): u \in C^{1}\left(S^{n}\right) \backslash\{0\}\right\}
$$

is achieved.

The main idea in the proof is to show that there is a minimizing sequence which strongly converges (in $L^{p_{*}}$ sense) to a nonzero function. Let

$$
\begin{aligned}
k(n, p) & =\pi^{-1 / 2} n^{-1 / p}\left(\frac{p-1}{n-p}\right)^{1-1 / p}\left\{\frac{\Gamma(1+n / 2) \Gamma(n)}{\Gamma(n / p) \Gamma(1+n-n / p)}\right\}^{1 / n} \\
& =\inf _{u \in C_{0}^{1}(\mathbb{R}) \backslash\{0\}} \frac{\left(\int_{\mathbb{R}^{n}}|\nabla u|^{p}\right)^{1 / p}}{\left(\int_{\mathbb{R}^{n}}|u|^{p_{*}}\right)^{1 / p_{*}}} ;
\end{aligned}
$$

and $k(n, 1)=\lim _{p \rightarrow 1+} k(n, p)=\pi^{-1 / 2} n^{-1}\{\Gamma(1+n / 2)\}^{1 / n}$. If one can prove that

$$
P_{\mathrm{I}}\left(S^{n}, p\right)<\frac{1}{k^{p}(n, p)},
$$

the convergence of a minimizing sequence will follow from some standard arguments. However, for general $p$ such a strict inequality may not be true. For example, from Bernstein inequality on $S^{2}$ one can prove (see more details in $[9])$ that

$$
\inf _{u \in B V\left(S^{2}\right) \backslash\{0\}} \frac{\int_{S^{2}}|\nabla u|}{\left(\int_{S^{2}}\left|u-u_{A}\right|^{n /(n-1)}\right)^{(n-1) / n}}=\frac{1}{k(2,1)}=2 \sqrt{\pi} .
$$

Nevertheless, by choosing a suitable (but standard) test function we can show the following:

Theorem 1.2. Let $\left(M^{n}, g\right)$ be a n-dimensional compact Riemannian manifold without boundary embedded in $R^{n+1}$. If $p \in(n /(n-1),(1+\sqrt{1+8 n}) / 4)$ for $n \geq 4$, then

$$
P_{\mathrm{I}}\left(M^{n}, p\right):=\inf _{u \in C^{1} \backslash\{0\}} \frac{\int_{M^{n}}\left|\nabla_{g} u\right|^{p}}{\left(\int_{M^{n}}\left|u-u_{a}\right|^{p_{*}}\right)^{p / p_{*}}}<\frac{1}{k^{p}(n, p)},
$$

and the infimum is achieved at some $u_{0} \in C^{1}\left(M^{n}\right)$.

The case of $p \in(n /(n-1),(1+\sqrt{1+8 n}) / 4)$ in Theorem 1.1 is obviously covered by Theorem 1.2. It is unclear whether the strict inequality (1.4) is still true for $p \geq(1+\sqrt{1+8 n}) / 4$ or not. On the other hand, one may check that if $n=3$ inequality (1.4) holds for $p=1$, thus it is true for $p \in\left[1,1+\delta_{0}\right.$ ) for some positive number $\delta_{0}$. Unfortunately, we have no information about this $\delta_{0}$. We may guess that the strict inequality (1.4) holds for all $p \in[1, n)$ and $n \geq 2$ except the case of $p=1$ and $n=2$. Even though we do not know whether (1.4) holds for general $p$ or not, however, if we return to the special manifold $S^{n}$ and change the constraint on $u$ slightly, we obtain the 
following result for all $p \in(1, n)$. For convenience, throughout the paper we denote

$$
H_{a}\left(S^{n}\right):=\left\{u \in C^{1}\left(S^{n}\right): \int_{S^{n}}|u|^{p_{*}-2} u=0\right\} .
$$

Theorem 1.3. If $1<p<n$, then

$$
P_{\mathrm{II}}\left(S^{n}, p\right):=\inf _{u \in H_{a}\left(S^{n}\right) \backslash\{0\}} \frac{\int_{S^{n}}|\nabla u|^{p}}{\left(\int_{S^{n}}|u|^{p_{*}}\right)^{p / p_{*}}},
$$

is achieved at some $u_{0} \in H_{a}\left(S^{n}\right)$.

It shall be pointed out that in the case of $n=2$ and $p=1$ we have $p_{*}-2=0$. Therefore the constraints on $u$ in Theorem 1.3 is the same as that in Theorem 1.1.

Based on the symmetrization result on $S^{n}$ (see, for example, Baernstein $[2])$, we can assume that there is a minimizing sequence depending only on one variable. Amazingly, the case of $p<2 n /(n+1)$ in Theorem 1.1 can be handled in the same spirit as that in the proof of Theorem 1.3 (note that this upper bound of $p$ matches the lower bound of $p$ in Theorem 1.2 perfectly).

We make a final remark in this introduction. When the manifold is a sphere with the standard metric, if one can show that there is an antisymmetric minimizing sequence $u_{k}$ (that is, $u\left(x^{\prime}, x_{n}\right)=-u\left(x^{\prime},-x_{n}\right)$ ) to $\inf I_{p}(u)$, one easily obtains (1.3) and Theorem 1.1 follows. Unfortunately, such an expectation may not be realized in practice, given the fact that there are the extremal functions for $\inf I_{1}(u)$ on $S^{2}$ which are not antisymmetric. We refer to [4] for some related issues on the symmetric properties of the extremal functions.

We organize the paper as follows: In Section 2, we prove Theorem 1.3; in Section 3, we prove Theorem 1.1 and Theorem 1.2.

\section{Proof of Theorem 1.3.}

For a small positive parameter $0<\epsilon \ll 1$, we define $q_{\epsilon}=p_{*}-\epsilon$,

$$
H_{a, \epsilon}\left(S^{n}\right):=\left\{u \in C^{1}\left(S^{n}\right): \int_{S^{n}}|u|^{q_{\epsilon}-2} u=0\right\},
$$

and

$$
S_{\epsilon}:=\inf _{u \in H_{a, \epsilon}\left(S^{n}\right) \backslash\{0\}} J_{\epsilon}(u):=\inf _{u \in H_{a, \epsilon}\left(S^{n}\right) \backslash\{0\}} \frac{\int_{S^{n}}|\nabla u|^{p}}{\left(\int_{S^{n}}|u|^{q_{\epsilon}}\right)^{p / q_{\epsilon}}} .
$$

Let $\left(\alpha_{1}, \ldots, \alpha_{n-1}, \theta\right)$ be the spherical coordinates of $S^{n}$, where $0 \leq \alpha_{i}<$ $2 \pi, \quad-\frac{\pi}{2} \leq \theta<\frac{\pi}{2}$. Standard variational method shows that $\inf J_{\epsilon}(u)$ is attained in $H_{a, \epsilon}\left(S^{n}\right)$. Further, the symmetrization argument (see, e.g., Baerstein $[2])$ yields that the extremal function $u_{\epsilon}(x)$ only depends on $\theta$ and is a 
monotonically non-decreasing function of $\theta$. We can normalize $u_{\epsilon}$ such that

$$
\int_{S^{n}}\left|u_{\epsilon}\right|^{q_{\epsilon}}=1
$$

Thus, $u_{\epsilon}$ satisfies the following equation:

$$
\left\{\begin{array}{l}
\nabla\left(\left|\nabla u_{\epsilon}\right|^{p-2} \nabla u_{\epsilon}\right)+S_{\epsilon}\left|u_{\epsilon}\right|^{q_{\epsilon}-2} u_{\epsilon}=0 \quad \text { in } S^{n} \\
d u_{\epsilon}(\theta) / d \theta \geq 0 .
\end{array}\right.
$$

If $\left\|u_{\epsilon}\right\|_{L^{\infty}} \leq C$, then from the elliptic estimates (see, for example, [5]) we know that $\left\|u_{\epsilon}\right\|_{C^{1, \alpha}} \leq C$ for some $\alpha \in(0,1)$, and conclude that up to a subsequence of $\epsilon, u_{\epsilon} \rightarrow u_{0}$ in $C^{1, \alpha}$ as $\epsilon \rightarrow 0$, where $u_{0}$ is the minimizer of $J_{0}$. Hence Theorem 1.3 is proved. So we shall focus on ruling out the case: Up to a subsequence of $\epsilon$,

$$
\left\|u_{\epsilon}\right\|_{L^{\infty}} \rightarrow \infty \quad \text { as } \epsilon \rightarrow 0 .
$$

We denote $\theta_{\epsilon}$ as the zero point of $u_{\epsilon}$, and assume that $\theta_{\epsilon} \rightarrow \theta_{0}$ (up to a subsequence of $\epsilon$ ).

Further, without loss of generality we can assume that

$$
\begin{cases}u_{\epsilon}(\theta)<0 & \text { for }-\frac{\pi}{2} \leq \theta<\theta_{\epsilon} \\ u_{\epsilon}(\theta)>0 & \text { for } \theta_{\epsilon}<\theta \leq \frac{\pi}{2},\end{cases}
$$

and $u_{\epsilon}(\pi / 2)=\max u_{\epsilon}(\theta)=\left\|u_{\epsilon}\right\|_{L^{\infty}}$.

Proposition 2.1. Given $\delta>0$, for any $\theta<\pi / 2-\delta$,

$$
u_{\epsilon}(\theta) \rightarrow 0 \quad \text { uniformly as } \epsilon \rightarrow 0 \text {. }
$$

Proof. The proposition can be proved via the following standard two steps:

First of all we claim that

$$
{\underline{\lim _{\epsilon \rightarrow 0}}}_{\epsilon} \leq \frac{1}{k^{p}(n, p)} .
$$

We relegate the proof of this inequality to the end of this paper.

Then we have the following concentration phenomena: For any fixed $\delta>$ 0 ,

$$
\liminf _{\epsilon \rightarrow 0} \int_{S^{n} \cap\{\theta>\pi / 2-\delta\}} u_{\epsilon}^{q_{\epsilon}}=1 .
$$

As a consequence, one can obtain Proposition 2.1. We refer readers to, for example, [7] for more details.

Now, for any $\tau \in(-\pi / 2, \pi / 2)$, we define $\mu_{\epsilon}=\max _{\theta \in[-\pi / 2, \tau]}\left|u_{\epsilon}(\theta)\right|$. From Proposition 2.1 we know that $\mu_{\epsilon} \rightarrow 0$ as $\epsilon \rightarrow 0$. Let $v_{\epsilon}(\theta)=-u_{\epsilon}(\theta) / \mu_{\epsilon}$. 
Then $v_{\epsilon}$ satisfies:

$$
\left\{\begin{array}{l}
\nabla\left(\left|\nabla v_{\epsilon}\right|^{p-2} \nabla v_{\epsilon}\right)+S_{\epsilon} \mu_{\epsilon}^{q_{\epsilon}-p} v_{\epsilon}^{q_{\epsilon}-1}=0 \text { in } S^{n} \cap\{-\pi / 2<\theta<\tau\} \\
0 \leq\left|v_{\epsilon}\right| \leq 1 \text { for }-\pi / 2<\theta<\tau .
\end{array}\right.
$$

It follows from the standard elliptic theory that $v_{\epsilon} \rightarrow v_{0}$ in any compact set of $\left\{x=\left(\alpha_{1}, \ldots, \alpha_{n-1}, \theta\right) \in S^{n} \mid-\pi / 2<\theta<\tau\right\}$, where $v_{0}$ satisfies:

$$
\begin{cases}\nabla\left(\left|\nabla v_{0}\right|^{p-2} \nabla v_{0}\right)=0 & \text { for }-\pi / 2<\theta<\tau \\ \max \left|v_{0}(\theta)\right|=1, \quad 0 \leq\left|v_{0}\right| \leq 1 & \text { for }-\pi / 2<\theta<\tau .\end{cases}
$$

Since $v_{0}(\theta)$ is monotonically non-decreasing in $\theta$, in both cases $\left(\tau>\theta_{0}\right.$ or $\left.\tau<\theta_{0}\right)$ we obtain a contradiction due to the maximum principle!

\section{Proof of Theorems 1.1 and 1.2 .}

Let us first establish Theorem 1.1 in the case of $p<2 n /(n+1)$. We follow the main stream in the proof of Theorem 1.3. For a small positive parameter $0<\epsilon \ll 1$, we define $q_{\epsilon}=p_{*}-\epsilon$, and

$$
Z_{\epsilon}:=\inf _{u \in H_{a, p_{*}}\left(S^{n}\right) \backslash\{0\}} I_{\epsilon}(u):=\inf _{u \in H_{a, p_{*}}\left(S^{n}\right) \backslash\{0\}} \frac{\int_{S^{n}}|\nabla u|^{p}}{\left(\int_{S^{n}}|u|^{q_{\epsilon}}\right)^{p / q_{\epsilon}}},
$$

where

$$
H_{a, p_{*}}\left(S^{n}\right)=\left\{u \in C^{1}\left(S^{n}\right): \int_{S^{n}} u=0\right\} .
$$

Standard variational method and the symmetrization argument show that $\inf I_{\epsilon}(u)$ is attained by $u_{\epsilon}(x)$ which depends only on $\theta$ and is a monotonically non-decreasing function of $\theta$. We normalize $u_{\epsilon}$ such that

$$
\int_{S^{n}}\left|u_{\epsilon}\right|^{q_{\epsilon}}=1
$$

If $\left\|u_{\epsilon}\right\|_{L^{\infty}}<\infty$, we are done. Otherwise, we assume, up to some subsequence of $\epsilon$, that

$$
\left\|u_{\epsilon}\right\|_{L^{\infty}} \rightarrow \infty \quad \text { as } \quad \epsilon \rightarrow 0 .
$$

Let $\theta_{\epsilon}$ be the zero point of $u_{\epsilon}$ and denote $c_{\epsilon}=\int_{S^{n}}\left|u_{\epsilon}\right|^{q_{\epsilon}-2} u_{\epsilon}$. Up to a further subsequence of $\epsilon$, we can assume that $\theta_{\epsilon} \rightarrow \theta_{0}$. It follows easily from Hölder inequality and (3.13) that

$$
\left|c_{\epsilon}\right| \leq C
$$

Without loss of generality, we can assume that

$$
\begin{cases}u_{\epsilon}(\theta)<0 & \text { for }-\frac{\pi}{2} \leq \theta<\theta_{\epsilon} \\ u_{\epsilon}(\theta)>0 & \text { for } \theta_{\epsilon}<\theta \leq \frac{\pi}{2}\end{cases}
$$


and $u_{\epsilon}(\pi / 2)=\max u_{\epsilon}(\theta)=\left\|u_{\epsilon}\right\|_{L^{\infty}}$. The Euler-Lagrange equation of $u_{\epsilon}$ is given by:

$$
\left\{\begin{array}{l}
\nabla\left(\left|\nabla u_{\epsilon}\right|^{p-2} \nabla u_{\epsilon}\right)+Z_{\epsilon}\left|u_{\epsilon}\right|^{q_{\epsilon}-2} u_{\epsilon}-Z_{\epsilon} c_{\epsilon}=0 \quad \text { in } \quad S^{n} \\
d u_{\epsilon}(\theta) / d \theta \geq 0 .
\end{array}\right.
$$

Easy to see that $\left|Z_{\epsilon} c_{\epsilon}\right|<C$. We define $\mu_{\epsilon}=-u_{\epsilon}\left(-\frac{\pi}{2}\right)$. As in the proof of Proposition 2.1, one can show that $\mu_{\epsilon} \rightarrow 0$.

If $\theta_{0}>-\pi / 2$, we let $v_{\epsilon}=-u_{\epsilon} / \mu_{\epsilon}$. Then $v_{\epsilon}$ satisfies

$$
\left\{\begin{array}{l}
\nabla\left(\left|\nabla v_{\epsilon}\right|^{p-2} \nabla v_{\epsilon}\right) \\
\quad+Z_{\epsilon} \mu_{\epsilon}^{q_{\epsilon}-p} v_{\epsilon}^{q_{\epsilon}-1}-Z_{\epsilon} c_{\epsilon} \mu_{\epsilon}^{1-p}=0 \quad \text { in } S^{n} \cap\left\{-\pi / 2<\theta<\theta_{\epsilon}\right\} \\
d u_{\epsilon}(\theta) / d \theta \geq 0, \\
\quad 0 \leq v_{\epsilon} \leq 1 \text { for }-\pi / 2<\theta<\theta_{\epsilon} .
\end{array}\right.
$$

For any fixed $p<2 n /(n+1)$, we choose positive constants $s$ and $t$ such that

$$
\left(q_{\epsilon}-1\right) t+s=q_{\epsilon}-1, \quad \text { and } \quad \frac{\left(q_{\epsilon}-1\right) t}{q_{\epsilon}}+s=1 .
$$

Then from Hölder inequality, we obtain:

$$
\begin{aligned}
\left|c_{\epsilon}\right| & \leq \int_{S^{n}}\left|u_{\epsilon}\right|^{q_{\epsilon}-1} \\
& \leq\left(\int_{S^{n}}\left|u_{\epsilon}\right|^{q_{\epsilon}}\right)^{\frac{\left(q_{\epsilon}-1\right) t}{q_{\epsilon}}} \cdot\left(\int_{S^{n}}\left|u_{\epsilon}\right|\right)^{s} \\
& =C \cdot\left(\int_{-\pi / 2}^{\theta_{\epsilon}}\left|u_{\epsilon}\right|\right)^{s} \quad\left(\text { using } \int_{S^{n}} u=0\right) \\
& \leq C_{1}\left|u_{\epsilon}\left(-\frac{\pi}{2}\right)\right|^{s} \\
& \leq C_{1} \mu_{\epsilon}^{s} .
\end{aligned}
$$

(3.17) implies $s=1 /\left(q_{\epsilon}-1\right)$. Using the fact that $p<2 n /(n+1)$, one can check that for small $\epsilon, s+1-p>0$. Thus it follows from the standard elliptic theory that $v_{\epsilon} \rightarrow v_{0}$ in $C^{1, \alpha}(K)$ for any compact set $K$ of $\{x=$ $\left.\left(\alpha_{1}, \ldots, \alpha_{n-1}, \theta\right) \in S^{n}:-\pi / 2<\theta<\theta_{0}\right\}$, where $v_{0}$ satisfies:

$$
\begin{cases}\nabla\left(\left|\nabla v_{0}\right|^{p-2} \nabla v_{0}\right)=0 & \text { for }-\pi / 2<\theta<\theta_{0} \\ v_{0}(-\pi / 2)=\max _{-\pi / 2 \leq \theta \leq \theta_{0}}\left|v_{0}(\theta)\right|=1, & \\ 0 \leq v_{0} \leq 1 & \text { for }-\pi / 2<\theta<\theta_{0} .\end{cases}
$$

We thus derive a contradiction due to the maximum principle. 
If $\theta_{0}=-\frac{\pi}{2}$, then for sufficiently small $\epsilon, \theta_{\epsilon}<0$, thus $u_{\epsilon}(\theta) \geq 0$ for $\theta \geq 0$. Therefore $\int_{S^{n} \cap\{-\pi / 2 \leq \theta \leq \pi / 4\}} u_{\epsilon} \leq 0$. It follows that

$$
\begin{aligned}
\left|u_{\epsilon}(-\pi / 2)\right| \cdot\left(\theta_{\epsilon}+\frac{\pi}{2}\right) & \geq C \int_{S^{n} \cap\left\{\theta_{\epsilon} \leq \theta \leq \pi / 4\right\}} u_{\epsilon} \\
& \geq C \int_{S^{n} \cap\{0 \leq \theta \leq \pi / 4\}} u_{\epsilon} \geq C u_{\epsilon}(0) .
\end{aligned}
$$

This yields that $\left|u_{\epsilon}(-\pi / 2)\right| \geq C u_{\epsilon}(0)$ for sufficiently small $\epsilon$. We then consider $v_{\epsilon}=-u_{\epsilon} / \mu_{\epsilon}$ in the lower hemisphere and obtain $v_{\epsilon} \rightarrow v_{0}$ in $C^{1, \alpha}(K)$ for any compact set $K$ of the lower hemisphere, where $v_{0}$ satisfies:

$$
\begin{cases}\nabla\left(\left|\nabla v_{0}\right|^{p-2} \nabla v_{0}\right)=0 & \text { for }-\pi / 2<\theta<0 \\ v_{0}(-\pi / 2)=\max _{-\pi / 2 \leq \theta \leq 0} v_{0}(\theta)=1, \quad 0 \leq\left|v_{0}\right| \leq 1 & \text { for }-\pi / 2<\theta<0 .\end{cases}
$$

Again this contradicts the maximum principle! We hereby complete the proof of Theorem 1.1 in the case of $p<2 n(n+1)$.

To prove Theorem 1.1 in the case of $p \geq 2 n(n+1)$, we only need to prove Theorem 1.2.

Proof of Theorem 1.2. We first establish Theorem 1.2 under the assumption that (1.4) holds.

We quote the following lemma from Aubin's book [1]:

Lemma 3.1. Given $\delta>0$, there is a constant $C(\delta)$ such that

$$
\left(\int_{M^{n}}|u|^{p_{*}} d v_{g}\right)^{p / p_{*}} \leq\left(k^{p}(n, p)+\delta\right) \int_{M^{n}}\left|\nabla_{g} u\right|^{p} d v_{g}+C(\delta) \int_{M^{n}}|u|^{p} d v_{g}
$$

holds for all $u \in W^{1, p}\left(M^{n}\right)$.

Let $\left\{u^{(m)}\right\}$ be a minimizing sequence with $\int_{M^{n}} u^{(m)} d v_{g}=0$ and

$$
\left\|u^{(m)}\right\|_{P_{*}, M^{n}}=\left(\int_{M^{n}}\left|u^{(m)}\right|^{p_{*}} d v_{g}\right)^{1 / p_{*}}=1 .
$$

Clearly, $\left\|u^{(m)}\right\|_{W^{1, p}\left(M^{n}\right)} \leq C$. After passing to a subsequence, $u^{(m)}$ converges weakly to some $\widetilde{u} \in W^{1, p}\left(M^{n}\right)$, and $u^{(m)}$ converges strongly to $\widetilde{u}$ in $L^{q}$ for any $q<p_{*}$. Thus $\int_{M^{n}} \widetilde{u} d v_{g}=0$. Due to Brezis-Lieb's lemma [3], it is not difficult to see that

$$
\int_{M^{n}}\left(\left|u^{(m)}\right|^{p_{*}}-\left|u^{(m)}-\widetilde{u}\right|^{p_{*}}\right) d v_{g}=\int_{M^{n}}|\widetilde{u}|^{p_{*}} d v_{g}+o(1),
$$

and consequently

$$
\int_{M^{n}}\left(\left|u^{(m)}\right|^{p_{*}}-\left|u^{(m)}-\widetilde{u}\right|^{p_{*}}\right) d v_{g} \leq 1+o(1), \quad \int_{M^{n}}|\widetilde{u}|^{p_{*}} d v_{g} \leq 1,
$$

where $o(1)$ denotes various quantity tending to zero as $m$ tends to $\infty$. 
Choose $\delta$ such that $\frac{1}{k^{p}(n, p)+\delta}-P_{\mathrm{I}}\left(M^{n}, p\right) \geq \delta / 2$. By the Sobolev embedding theorem and Lemma 3.1, we have:

$$
\begin{aligned}
& P_{\mathrm{I}}\left(M^{n}, p\right) \\
& =\int_{M^{n}}\left|\nabla_{g} u^{(m)}\right|^{p}+o(1) \\
& =\int_{M^{n}}\left|\nabla_{g}\left(u^{(m)}-\widetilde{u}\right)\right|^{p}+\int_{M^{n}}\left|\nabla_{g} \widetilde{u}\right|^{p}+o(1) \\
& =\int_{M^{n}} \mid \nabla\left(u^{(m)}-\left.\widetilde{u}\right|^{p}+\frac{C(\delta)}{k^{p}(n, p)+\delta} \int_{M^{n}}\left|u^{(m)}-\widetilde{u}\right|^{p}+\int_{M^{n}}\left|\nabla_{g} \widetilde{u}\right|^{p}+o(1)\right. \\
& \geq \frac{1}{k^{p}(n, p)+\delta}\left(\int_{M^{n}}\left|u^{(m)}-\widetilde{u}\right|^{p_{*}}\right)^{p / p_{*}}+P_{\mathrm{I}}\left(M^{n}, p\right)\left(\int_{M^{n}} \widetilde{u}^{p_{*}}\right)^{p / p_{*}}+o(1) \\
& \geq \frac{1}{k^{p}(n, p)+\delta} \int_{M^{n}}\left|u^{(m)}-\widetilde{u}\right|^{p_{*}}+P_{\mathrm{I}}\left(M^{n}, p\right) \int_{M^{n}} \widetilde{u}^{p_{*}}+o(1) \\
& =\left(\frac{1}{k^{p}(n, p)+\delta}-P_{\mathrm{I}}\left(M^{n}, p\right)\right) \int_{M^{n}}\left|u^{(m)}-\widetilde{u}\right|^{p_{*}}+P_{\mathrm{I}}\left(M^{n}, p\right)+o(1) .
\end{aligned}
$$

This yields that $\int_{M^{n}}\left|u^{(m)}-\widetilde{u}\right|^{p_{*}}=o(1)$. It follows easily that $\widetilde{u}$ is a minimizer. Theorem 1.2 is proved.

We are left to verify (1.4).

Proof of (1.4).

We follow closely the computations in Druet [6]. Since $M^{n}$ is embedded in $R^{n+1}$, there is a point $x_{0} \in M$ such that the scalar curvature at $x_{0}$ (denoted as $R_{g}\left(x_{0}\right)$ ) is positive. We choose a normal geodesic coordinates system near $x_{0}$. Let $r$ be the distance from point $x$ to $x_{0}$, we define, for small positive constants $\epsilon$ and $\delta$,

$$
u_{\epsilon}(x)=u_{\epsilon}(r)=\left(\frac{1}{\epsilon+r^{p /(p-1)}}\right)^{\frac{n}{p}-1} \cdot \varphi(r),
$$

where $\varphi(r)$ is a nonnegative cut-off function satisfying:

$$
\varphi(r) \begin{cases}=1 & \text { for } \quad r \leq \frac{\delta}{2} \\ \leq 1 & \text { for } \quad \frac{\delta}{2} \leq r \leq \delta, \\ =0 & \text { for } \quad r \geq \delta .\end{cases}
$$

Notice that in this system,

$$
d v_{g}=\left[1-\frac{1}{6} R_{i j}\left(x_{0}\right) x^{i} x^{j}+o_{r}(1)\left(r^{2}\right)\right] d x^{i} d x^{j},
$$

where and throughout this section we denote $o_{\gamma}(1)$ as the term tending to 0 as $\gamma \rightarrow 0$.

We divide our computation into four steps. 
Step 1. Estimate of $\int_{M^{n}} u_{\epsilon}^{p_{*}} d v_{g}$.

We have, from (3.18), that

$$
\begin{aligned}
& \int_{M^{n}} u_{\epsilon}^{p_{*}} d v_{g} \\
& \geq \int_{B_{\delta / 2}\left(x_{0}\right)}\left(\frac{1}{\epsilon+r^{p /(p-1)}}\right)^{\left(\frac{n}{p}-1\right) \cdot p_{*}} d v_{g} \\
& =\omega_{n-1} \int_{0}^{\delta / 2}\left(\epsilon+r^{p /(p-1)}\right)^{-n} r^{n-1} d r \\
& -\frac{\omega_{n-1} R_{g}\left(x_{0}\right)}{6 n}\left(1+o_{\delta}(1)\right) \int_{0}^{\delta / 2}\left(\epsilon+r^{p /(p-1)}\right)^{-n} r^{n+1} d r \\
& =\omega_{n-1} \epsilon^{-\frac{n}{p}} \int_{0}^{\frac{\delta}{2 \epsilon^{(p-1) / p}}}\left(1+\beta^{p /(p-1)}\right)^{-n} \\
& \cdot\left[\beta^{n-1}-\frac{R_{g}\left(x_{0}\right)}{6 n}\left(1+o_{\delta}(1)\right) \cdot \beta^{n+1} \cdot \epsilon^{2(p-1) / p}\right] d \beta,
\end{aligned}
$$

where $\omega_{n-1}$ is the surface area of $S^{n-1}$. For $p<\frac{n+2}{2}$ we know that $n+2-$ $p n /(p-1)<0$. Thus

$$
\int_{0}^{\frac{\delta}{2 \epsilon^{(p-1) / p}}}\left(1+\beta^{p /(p-1)}\right)^{-n} \cdot \beta^{n+1} d \beta \leq C .
$$

It follows that (we also use $n>2(p-1)$ )

$$
\begin{aligned}
& \omega_{n-1} \epsilon^{-n / p} \int_{0}^{\frac{\delta}{2 \epsilon^{(p-1) / p}}}\left(1+\beta^{p /(p-1)}\right)^{-n} \beta^{n-1} d \beta \\
& \geq D_{1} \cdot \epsilon^{-n / p}-C \delta^{-\frac{n}{p-1}} \epsilon^{n / p} \cdot \epsilon^{-n / p} \\
& =D_{1} \cdot \epsilon^{-n / p}-o_{\epsilon}(1) \delta^{-\frac{n}{p-1}} \epsilon^{\frac{-n+2(p-1)}{p}},
\end{aligned}
$$

where

$$
D_{1}=\omega_{n-1} \int_{0}^{\infty} \frac{\beta^{n-1}}{\left(1+\beta^{p /(p-1)}\right)^{n}} d \beta
$$

and

$$
\begin{aligned}
& \frac{\omega_{n-1} R_{g}\left(x_{0}\right) \epsilon^{-n / p}}{6 n}\left(1+o_{\delta}(1)\right) \int_{0}^{\frac{\delta}{2 \epsilon^{(p-1) / p}}}\left(1+\beta^{p /(p-1)}\right)^{-n} \beta^{n+1} \cdot \epsilon^{2(p-1) / p} d \beta \\
& \leq D_{2}\left(1+o_{\delta}(1)\right) \epsilon^{-n / p+2(p-1) / p}
\end{aligned}
$$

where

$$
D_{2}=\frac{\omega_{n-1} R_{g}\left(x_{0}\right) \epsilon^{-n / p}}{6 n} \int_{0}^{\infty} \frac{\beta^{n+1}}{\left(1+\beta^{p /(p-1)}\right)^{n}} d \beta
$$


Therefore, for $p<\frac{n+2}{2}$,

$$
\int_{M^{n}} u_{\epsilon}^{p_{*}} d v_{g} \geq D_{1} \epsilon^{-n / p}-\left(D_{2}+o_{\epsilon, \delta}(1)\right) \epsilon^{-n / p+2(p-1) / p},
$$

where $o_{\epsilon, \delta}(1)$ means that for any $\gamma>0$ there is a $\delta_{0}$ such that for $\delta<\delta_{0}$, $\liminf _{\epsilon \rightarrow 0} o_{\epsilon, \delta}(1)<\gamma$. Notice that $(1+\sqrt{1+8 n}) / 4<(n+2) / 2$ for $n \geq 2$.

Step 2. Estimate of $\int_{M}\left|\nabla u_{\epsilon}\right|^{p} d v_{g}$.

If we denote

$$
\tau(r)=\left(\epsilon+r^{p /(p-1)}\right)^{1-n / p},
$$

then

$$
\nabla u_{\epsilon}=\nabla \tau(r) \varphi(r)+\tau(r) \nabla \varphi(r)
$$

and

$$
\left|\nabla u_{\epsilon}\right|_{g}^{p} \leq|\varphi(r) \nabla \tau(r)|_{g}^{p}+\nu|\nabla \varphi(r) \tau(r)|_{g}^{p}+\mu|\nabla \varphi(r) \tau(r)|_{g} \cdot|\varphi(r) \nabla \tau(r)|_{g}^{p-1}
$$

for some positive constants $\nu$ and $\mu$.

Since $\nabla \varphi(r)=0$ for $0 \leq r \leq \delta / 2$, we know that

$$
\int_{M^{n}}\left(\nu|\nabla \varphi(r) \tau(r)|^{p}+\mu|\nabla \varphi(r) \tau(r)| \cdot|\nabla \tau(r)|^{p-1}\right) d v_{g} \leq C(\delta)
$$

for some constant $C(\delta)$ independent of $\epsilon$. On the other hand, noting that $u_{\epsilon}$ is radially symmetric and $g^{r r}=1$, we know that

$$
|\varphi(r) \nabla \tau(r)|_{g}^{p} \leq|\nabla \tau(r)|_{g}^{p}=\left(\frac{n-p}{p-1}\right)^{p} \cdot r^{\frac{p}{p-1}} \cdot\left(\epsilon+r^{p /(p-1)}\right)^{-n} .
$$

Therefore

$$
\begin{aligned}
& \int_{M^{n}}\left|\nabla u_{\epsilon}\right|^{p} d v_{g} \\
& \leq C(\delta)+\int_{M}|\nabla \tau(r)|^{p} d v_{g} \\
& \leq C(\delta)+\omega_{n-1} \int_{0}^{\delta}\left(\frac{n-p}{p-1}\right)^{p} \cdot r^{p /(p-1)}\left(\epsilon+r^{p /(p-1)}\right)^{-n} \cdot r^{n-1} d r \\
& \quad-\frac{\omega_{n-1} R_{g}\left(x_{0}\right)}{6 n}\left(1+o_{\delta}(1)\right) \int_{0}^{\delta}\left(\frac{n-p}{p-1}\right)^{p} \cdot r^{p /(p-1)}\left(\epsilon+r^{p /(p-1)}\right)^{-n} r^{n+1} d r .
\end{aligned}
$$

One can easily check:

$$
\begin{aligned}
& \omega_{n-1}\left(\frac{n-p}{p-1}\right)^{p} \int_{0}^{\delta}\left(\epsilon+r^{p /(p-1)}\right)^{-n} \cdot r^{p /(p-1)+n-1} d r \\
& =\omega_{n-1}\left(\frac{n-p}{p-1}\right)^{p} \epsilon^{1-\frac{n}{p}} \cdot \int_{0}^{\delta \epsilon^{\frac{1-p}{p}}}\left(1+\beta^{p /(p-1)}\right)^{-n} \beta^{p /(p-1)+n-1} d \beta \\
& \leq\left(E_{1}+C \delta^{\frac{p-n}{p-1}} \cdot \epsilon^{\frac{n-p}{p}}\right) \epsilon^{1-\frac{n}{p}},
\end{aligned}
$$


where

$$
E_{1}=\omega_{n-1}\left(\frac{n-p}{p-1}\right)^{p} \int_{0}^{\infty}\left(1+\beta^{p /(p-1)}\right)^{-n} \beta^{p /(p-1)+n-1} d \beta .
$$

Note that $p /(p-1)+n-1>-1$, and $p /(p-1)+n-p n /(p-1)=$ $(p-n) /(p-1)<0$, thus $E_{1}<\infty$. Also for $p<(n+2) / 3$,

$$
\begin{aligned}
& \frac{\omega_{n-1} R_{g}\left(x_{0}\right)}{6 n}\left(\frac{n-p}{p-1}\right)^{p} \int_{0}^{\delta}\left(\epsilon+r^{p /(p-1)}\right)^{-n} \cdot r^{p /(p-1)+n+1} d r \\
& =\frac{\omega_{n-1} R_{g}\left(x_{0}\right)}{6 n}\left(\frac{n-p}{p-1}\right)^{p} \epsilon^{\frac{3 p-2-n}{p}} \cdot \int_{0}^{\delta \epsilon^{\frac{1-p}{p}}}\left(1+\beta^{p /(p-1)}\right)^{-n} \beta^{p /(p-1)+n+1} d \beta \\
& \leq\left(E_{2}+C \delta^{\frac{p-n}{p-1}+2} \cdot \epsilon^{\frac{n+2-3 p}{p}}\right) \epsilon^{1-\frac{n}{p}+\frac{2(p-1)}{p}},
\end{aligned}
$$

where

$$
E_{2}=\frac{\omega_{n-1} R_{g}\left(x_{0}\right)}{6 n}\left(\frac{n-p}{p-1}\right)^{p} \int_{0}^{\infty}\left(1+\beta^{p /(p-1)}\right)^{-n} \beta^{p /(p-1)+n+1} d \beta<\infty .
$$

For $p<(n+2) / 3$, we have $(n-p) / p>2(p-1) / p$. Thus,

$$
\int_{M^{n}}\left|\nabla u_{\epsilon}\right|^{p} d v_{g} \leq C(\delta)+E_{1} \epsilon^{1-\frac{n}{p}}-E_{2}\left(1+o_{\epsilon, \delta}(1)\right) \epsilon^{1-\frac{n}{p}+\frac{2(p-1)}{p}} .
$$

We also note that $(1+\sqrt{1+8 n}) / 4<(n+2) / 3$ for $n \geq 2$.

Step 3. Estimates of $\int_{M^{n}} u_{\epsilon}$.

$$
\begin{aligned}
\int_{M^{n}} u_{\epsilon} & \leq \omega_{n-1} \int_{0}^{\delta}\left(\epsilon+r^{p /(p-1)}\right)^{1-n / p} r^{n-1} d r \\
& \leq C_{1} \epsilon^{1-\frac{n}{p}+\frac{n(p-1)}{p}} \cdot \int_{0}^{\delta \epsilon^{\frac{1-p}{p}}}\left(1+\beta^{p /(p-1)}\right)^{1-\frac{n}{p}} \beta^{n-1} d \beta \\
& \leq\left\{\begin{array}{lll}
C(\delta) \epsilon^{\frac{n p+p-2 n}{p}} & \text { if } n p+p-2 n<0 \\
C(\delta) \ln \left(\delta \epsilon^{\frac{1-p}{p}}+C\right) & \text { if } n p+p-2 n=0 \\
C(\delta) & \text { if } & n p+p-2 n>0 .
\end{array}\right.
\end{aligned}
$$

Step 4. Define $w_{\epsilon}=u_{\epsilon}-\int_{M^{n}} u_{\epsilon}$. Then $\int_{M^{n}}\left|\nabla w_{\epsilon}\right|^{p}=\int_{M^{n}}\left|\nabla u_{\epsilon}\right|^{p}$. From Minkowski inequality, we know

$$
\left|\left(\int_{M^{n}}\left|w_{\epsilon}\right|^{p_{*}}\right)^{1 / p_{*}}-\left(\int_{M^{n}}\left|u_{\epsilon}\right|^{p_{*}}\right)^{1 / p_{*}}\right| \leq \operatorname{vol}^{1 / p_{*}}\left(M^{n}\right) \cdot \int_{M^{n}}\left|u_{\epsilon}\right| .
$$


From (3.20) we have

$$
\begin{aligned}
& \left(\int_{M^{n}}\left|u_{\epsilon}\right|^{p_{*}}\right)^{1 / p_{*}} \\
& \geq\left(D_{1} \epsilon^{-n / p}-\left(D_{2}+o_{\epsilon, \delta}(1)\right) \epsilon^{-n / p+2(p-1) / p}\right)^{1 / p_{*}} \\
& =D_{1}^{1 / p_{*}} \epsilon^{-\frac{n}{p p_{*}}}-\left(\frac{D_{1}^{1 / p_{*}} D_{2}}{D_{1} p_{*}}+o_{\epsilon, \delta}(1)\right) \epsilon^{-\frac{n}{p p_{*}}+\frac{2(p-1)}{p}} .
\end{aligned}
$$

If $p \geq 2 n /(n+1)$ (i.e., $n p+p-2 n \geq 0)$ and

$$
-\frac{n}{p p_{*}}+\frac{2(p-1)}{p}<0
$$

we obtain from (3.22) that

$$
\left(\int_{M^{n}}\left|w_{\epsilon}\right|^{p_{*}}\right)^{1 / p_{*}} \geq D_{1}^{1 / p_{*}} \epsilon^{-\frac{n}{p p_{*}}}-\left(\frac{D_{1}^{1 / p_{*}} D_{2}}{D_{1} p_{*}}+o_{\epsilon, \delta}(1)\right) \epsilon^{-\frac{n}{p p_{*}}+\frac{2(p-1)}{p}} .
$$

This is the place where we need the condition $p<(1+\sqrt{1+8 n}) / 4$ to guaranteer that (3.24) holds.

On the other hand, if the dimension $n \geq 4$, for any $p \in(n /(n-1), 2 n /(n+$ 1)), one can check:

$$
-\frac{n}{p p_{*}}+\frac{2(p-1)}{p}<\frac{n p+p-2 n}{p}<0 .
$$

From (3.22) and (3.23) we know that (3.25) still holds. From (3.25) and (3.21) one can derive that for some small enough $\delta$ and $\epsilon$,

$$
\begin{aligned}
\frac{\int_{M^{n}}\left|\nabla w_{\epsilon}\right|^{p}}{\left(\int_{M^{n}}\left|w_{\epsilon}\right|^{p_{*}}\right)^{p / p_{*}}} & \leq \frac{C(\delta)+E_{1} \epsilon^{1-\frac{n}{p}}-E_{2}\left(1+o_{\epsilon, \delta}(1)\right) \epsilon^{1-\frac{n}{p}+\frac{2(p-1)}{p}}}{D_{1}^{p / p_{*}} \epsilon^{-\frac{n}{p_{*}}}-\left(\frac{p D_{1}^{p / p *} D_{2}}{p_{*} D_{1}}+o(1)\right) \epsilon^{-\frac{n}{p_{*}}+\frac{2(p-1)}{p}}} \\
& <\frac{1}{k^{p}(n, p)} .
\end{aligned}
$$

More details about the derivation of the second inequality in the above can be found in Druet's paper [6]. We hereby complete the Proof of (1.4). 
Now we return to the proof of (2.9). We use the same test function. Similar to Step 3, we have

$$
\begin{aligned}
& \int_{M^{n}}\left|u_{\epsilon}\right|^{p_{*}-2} u_{\epsilon} \\
& \leq \omega_{n-1} \int_{0}^{\delta}\left(\epsilon+r^{p /(p-1)}\right)^{-(n p-n+p) / p} r^{n-1} d r \\
& \leq C_{1} \epsilon^{-\frac{n p-n+p}{p}+\frac{n(p-1)}{p}} \cdot \int_{0}^{\delta \epsilon \frac{1-p}{p}}\left(1+\beta^{p /(p-1)}\right)^{-(n p-n+p) / p} \beta^{n-1} d \beta \\
& \leq C \epsilon^{-1} .
\end{aligned}
$$

We choose $C_{\epsilon}$ such that for $v_{\epsilon}=u_{\epsilon}-C_{\epsilon}$,

$$
\int_{M^{n}}\left|v_{\epsilon}\right|^{p_{*}-2} v_{\epsilon} d v_{g}=0
$$

Thus

$$
\begin{aligned}
& \int_{\left\{x \in M^{n}: u_{\epsilon} \geq C_{\epsilon}\right\}}\left|u_{\epsilon}-C_{\epsilon}\right|^{p_{*}-2}\left(u_{\epsilon}-C_{\epsilon}\right) d v_{g} \\
& =-\int_{\left\{x \in M^{n}: u_{\epsilon}<C_{\epsilon}\right\}}\left|u_{\epsilon}-C_{\epsilon}\right|^{p_{*}-2}\left(u_{\epsilon}-C_{\epsilon}\right) d v_{g} .
\end{aligned}
$$

This implies that $C_{\epsilon} \geq 0$. It follows that

$$
\begin{aligned}
\int_{M^{n}}\left|u_{\epsilon}-C_{\epsilon}\right|^{p_{*}-1} d v_{g} & =2 \int_{\left\{x \in M^{n}: u_{\epsilon} \geq C_{\epsilon}\right\}}\left|u_{\epsilon}-C_{\epsilon}\right|^{p_{*}-1} d v_{g} \\
& \leq 2 \int_{M^{n}}\left|u_{\epsilon}\right|^{p_{*}-1} d v_{g} \leq 2 C \epsilon^{-1} .
\end{aligned}
$$

But for any fixed $p_{*}$, there are two positive constants $a$ and $b$ depending only on $p_{*}$ such that

$$
(x-1)^{p_{*}-1} \geq a x^{p_{*}-1}-b, \quad \forall x \geq 1 .
$$

Applying the above in (3.26) we obtain that $C_{\epsilon} \leq C \epsilon^{-1 /\left(p_{*}-1\right)}$. Using Minkowski inequality, we have

$$
\left(\int_{M^{n}}\left|u_{\epsilon}\right|^{p_{*}}\right)^{1 / p_{*}}-C \cdot C_{\epsilon} \leq\left(\int_{M^{n}}\left|v_{\epsilon}\right|^{p_{*}}\right)^{1 / p_{*}} \leq\left(\int_{M^{n}}\left|u_{\epsilon}\right|^{p_{*}}\right)^{1 / p_{*}}+C \cdot C_{\epsilon} .
$$

For $p \in(1, n)$, we have $-1 /\left(p_{*}-1\right)>-n /\left(p p_{*}\right)$. Similar to the computation in (3.20) we know that

$$
\left(\int_{M^{n}}\left|u_{\epsilon}\right|^{p_{*}}\right)^{1 / p_{*}}=\left(1+o_{\epsilon}(1)\right) D_{1}^{1 / p_{*}} \epsilon^{-n /\left(p p_{*}\right)}, \quad \forall p \in(1, n) .
$$


Thus $C_{\epsilon}=o_{\epsilon}(1)\left(\int_{M^{n}}\left|u_{\epsilon}\right|^{p_{*}}\right)^{1 / p_{*}}$, and

$$
\left(\int_{M^{n}}\left|v_{\epsilon}\right|^{p_{*}}\right)^{1 / p_{*}}=\left(1+o_{\epsilon}(1)\right)\left(\int_{M^{n}}\left|u_{\epsilon}\right|^{p_{*}}\right)^{1 / p_{*}} .
$$

It follows that

$$
\varliminf_{\epsilon \rightarrow 0} S_{\epsilon} \leq S_{0} \leq \lim _{\epsilon \rightarrow 0} \frac{\int_{M^{n}}\left|\nabla_{g} v_{\epsilon}\right|^{p}}{\left(\int_{M^{n}}\left|v_{\epsilon}\right|^{p_{*}}\right)^{p / p_{*}}}=\lim _{\epsilon \rightarrow 0} \frac{\int_{M^{n}}\left|\nabla_{g} u_{\epsilon}\right|^{p}}{\left(\int_{M^{n}}\left|u_{\epsilon}\right|^{p_{*}}\right)^{p / p_{*}}} \leq \frac{1}{k^{p}(n, p)} .
$$

Acknowledgments. This paper should be a joint work with Elliott $\mathrm{H}$. Lieb. Most ideas came out from some stimulative discussions with him; the author respects his decision with admiration that he will not put his name on this paper, and would like to dedicate this paper to him. The author thanks A. Baernstein and E. Hebey for some useful discussions. This work has been done while the author was visiting Princeton University, and was partially supported by the American Mathematical Society Centennial fellowship. He also would like to thank A. Chang, P. Yang and Mathematics Department of Princeton University for their hospitality during his visit.

\section{References}

[1] T. Aubin, Nonlinear Analysis on Manifolds. Monge-Ampere Equations, Springer-Verlag, Berlin, 1982, Grundlehern Math. Wiss., 252, MR 0681859, Zbl 0512.53044.

[2] A. Baernstein, A unified approach to symmetrization, in 'Partial Differential Equations of Elliptic type. Sympos. Math. XXXV', edited by A. Alvino, etc., Cambridge Univ. Press, Cambridge, 1994, 47-91, MR 1297773, Zbl 0830.35005.

[3] H. Brezis and E. Lieb, A relation between pointwise convergence of functions and convergence of functionals, Proc. Amer. Math. Soc., 88(3) (1983), 486-490, MR 0699419, Zbl 0526.46037.

[4] B. Dacorogna, W. Gangbo and N. Subia, Sur une généralisation de I'inǵalité de Wirtinger, Ann. Inst. H. Poincaré Anal. Non Linéaire, 9(1) (1992), 29-50, MR 1151466, Zbl 0764.49009.

[5] E. DiBenedetto, $C^{1+\alpha}$ local regularity of weak solutions of degenerate elliptic equations, Nonlinear Anal., 7(8) (1983), 827-850, MR 0709038, Zbl 0539.35027.

[6] O. Druet, Optimal Sobolev inequalities of arbitrary order on compact Riemannian manifolds, J. Functional Analysis, 159 (1998), 217-242, MR 1654123, Zbl 0923.46035.

[7] N. Ghoussoub, C. Gui and M. Zhu, On a singularly perturbed Neumann problem with the critical exponent, Comm. Partial Differential Equations, 26(11-12) (2001), 19291946, MR 1876408, Zbl 0997.35021.

[8] E. Hebey, Sobolev Spaces on Riemannian Manifolds, Lecture Notes in Mathematics, 1635, Springer, 1996, MR 1481970, Zbl 0866.58068. 
ON THE EXTREMAL FUNCTIONS OF SOBOLEV-POINCARÉ INEQUALITY 199

[9] M. Zhu, Sharp Poincaré-Sobolev inequalities and the shortest length of simple closed geodesics on a topological two sphere, preprint.

Received February 6, 2003 and revised June 2, 2003.

Department of Mathematics

UNIVERSITY OF OKLAHOMA

NORMAN, OK 73019

E-mail address: mzhu@aftermath.math.ou.edu 